\title{
The Impact of Age in Acute Type A Aortic Dissection: a Retrospective Study
}

\section{Junxia Wang}

Department of Cardiothoracic Surgery, Affiliated Drum Tower Hospital, Medical School of Nanjing University Yunxing Xue

Department of Cardiothoracic Surgery, Affiliated Drum Tower Hospital, Medical School of Nanjing University

Xiyu Zhu

Department of Cardiothoracic Surgery, Affiliated Drum Tower Hospital, Medical School of Nanjing University

Hoshun Chong

Department of Cardiothoracic Surgery, Affiliated Drum Tower Hospital, Medical School of Nanjing University

Zhong Chen

Department of Cardiothoracic Surgery, Affiliated Drum Tower Hospital, Medical School of Nanjing University

Qing Zhou

Department of Cardiothoracic Surgery, Affiliated Drum Tower Hospital, Medical School of Nanjing University

Jason Zhensheng Qu

Massachusetts General Hospital Department of Anesthesia Critical Care and Pain Medicine

Dongjin Wang ( $\nabla$ dongjinwang_gl@163.com)

The Affiliated Drum Tower Hospital of Nanjing University Medical School https://orcid.org/0000-0002-6835-2526

\section{Research article}

Keywords: Aortic dissection, age, surgical therapy

Posted Date: October 18th, 2021

DOI: https://doi.org/10.21203/rs.3.rs-970708/v1

License: (ㄷ) (i) This work is licensed under a Creative Commons Attribution 4.0 International License. Read Full License

Version of Record: A version of this preprint was published at Journal of Cardiothoracic Surgery on March 19th, 2022. See the published version at https://doi.org/10.1186/s13019-022-01785-y. 


\section{Abstract}

Background: Acute type A aortic dissection (aTAAD) is a lethal disease and age is an important risk factor for outcomes. This retrospective study was to analyze the impact of age stratification in aTAAD, and to provide clues for surgeons when they make choices of therapy strategies.

Methods: From January 2011 to December 2019, 1092 aTAAD patients from Nanjing Drum Tower Hospital received surgical therapy. Patients were divided into 7 groups according to every ten-year interval (20s-80s). The differences between the groups were analyzed in terms of the baseline preoperative conditions, surgical methods and postoperative outcomes of patients of different age groups. During a median follow-up term of 17 months, the survival rates were compared among 7 groups through Kaplan-Meier analysis.

Results: The median age was 52.0 years old in whole cohort. The multiple comorbidities were more common in old age groups (60s, 70s, $80 \mathrm{~s})$, while the 20s group patients had the highest proportion of Marfan syndrome (28.1\%). Preoperative hypotension was highest in 80 s $(16.7 \%, P=0.038)$. Young age groups (20s-60s) had a higher rate of root replacement and total arch replacement, which led to a longer duration of operation and hypothermic circulation arrest. The overall mortality was $14.1 \%$, the tendency of mortality was increased with age except 20 s group (33.3\% in 80 s, $\mathrm{P}=0.016$ ). The postoperative morbidity of gastrointestinal bleeding and bowel ischemia were $16.7 \%$ and $11.1 \%$ in 80 s group.

Conclusions: Age is a major impact factor for aTAAD surgery. Old patients presented more comorbidities before surgery, the mortality and complications rate were significantly higher even with less invasive and conservative surgical therapy. But the favorable long term survival indicated that the simple or less extensive arch repair is the preferred surgery for patients over 70 years old.

\section{Background}

Age has shown to be a strong independent impact factor of outcomes in acute type A aortic dissection (aTAAD), while treatment strategies and surgical methods differentiate according to age stratification. Old age has long been thought to increase the risk of surgery, which likely explains the higher rate of medical therapy especially in patients older than 70 years old [1]. As the population ages, surgical techniques and perioperative management continue to improve, advanced age is no longer a major deterrent of surgical repair of aTAAD albeit conservative therapy is chosen by patients and physicians[2, 3]. Studies have shown that the average age of patients with aTAAD is 55 years old in China, a decade younger than that in the western countries $[4,5]$. So an extensive one-stage surgery, total arch replacement and frozen elephant trunk, has become the preferred surgical strategy in China to avoid reintervention[6, 7]. There are also reports on one-stage total aortic arch replacement in other counties.

However, as the number of patients with aTAAD increases in age groups in China, especially in the groups of 60-80 years of age, the long-term survival of this extensive surgery is largely unknown. Should age be factored in the consideration before such an extensive surgery be planed? The age-stratified clinical characteristics, treatment strategies, and outcomes in Chinese patients are not yet known. The aim of this retrospective study is to investigate the clinical characteristics, treatment strategy and surgical outcomes of aTAAD in different age stratification groups in our center.

\section{Methods}

\subsection{Patients}

Between January 2011 and December 2019, a total of 1174 patients with aTAAD were admitted to Nanjing Drum Tower Hospital (NDTH). Patients were divided into seven groups according to every ten-year interval (20s-80s). Diagnosis of aTAAD was confirmed by computed tomographic angiography (CTA) scanning within two weeks after the onset of symptoms.

All clinical data were collected prospectively by admission and during the in-hospital stay. We retrieved the data retrospectively by review of hospital records. The study was conducted in accordance with the Declaration of Helsinki (as revised in 2013). The current study was approved by the institutional review board of Nanjing Drum Tower Hospital (2020-185-01).

\subsection{Treatment}

All patients diagnosed with aTAAD were transferred to cardiac surgery intensive care unit and optimal medical therapy was initiated. Open surgery was recommended for all patients, but for patients with advanced age, dissection associated organ malperfusion or family refusal, medical therapy was the treatment of choice. The patients received open surgery underwent general anesthesia via a standard median sternotomy after signing the informed consents. Cardiopulmonary bypass (CPB) was initiated with femoral artery or axillary artery arterial cannulation and right atrium or superior/inferior venous cannulation. Deep or mild hypothermic circulatory arrest (HCA) was used in all patients. Selective antegrade or retrograde cerebral perfusion was applied for brain protection during the period of HCA at operating surgeon's choice. The distal aortic arch surgical strategy included partial arch replacement, total arch replacement with or without frozen elephant trunk (Microport Corp.Ltd, Shanghai, China) and arch stent (Yuhengjia Sci Tech Corp.Ltd, Beijing, China) based on the pathological involvement of the aortic arch[7-9]. After finishing the distal repair, the rewarming stage begun as the proximal part of aorta or root was being reconstructed and the patients were weaned off CPB. Bentall procedure or root reinforcement reconstruction was applied based on the anatomic indications[10,11]. The patients were transferred to the floor after recovering in cardiac surgery ICU, and discharged from the hospital per institution protocol. 


\subsection{Statistical analysis}

Statistical analysis was performed with SPSS 26.0 (IBM Corp. Released 2019. IBM SPSS Statistics for Macintosh, Version 26.0. Armonk, NY: IBM Corp.). Descriptive statistics were used to describe patient characteristics throughout the study. Means and standard deviations were presented for normally distributed continuous variables whereas median and the interquartile ranges were computed to describe non-normally distributed continuous data. Categorical data are presented as frequency distributions and simple percentages. Between group differences were analyzed using a Student's t-test,Kruskal-Wallis H test or Mann-Whitney U-test for continuous variables and a Chi-square or Fisher's exact test for categorical variables. The survival curve was draw using Kaplan-Meier method and compared using the log-rank test. The median follow-up time was calculated with reverse Kaplan-Meier method. Statistical significance was considered when $\mathrm{P}<0.05$.

\section{Results}

\subsection{Demographics and Preoperative characteristics}

Eighty-two of the 1174 aTAAD patients chose medical management that was chosen by $30.8 \%$ of patients in 80 years group due to rupture of the dissection (Figure $1 \mathrm{a} / \mathrm{b}$ ). The patients aged $40-60$ years constituted the largest proportion of patients $(71.2 \%)$ and the youngest 20 s (2.9\%) and oldest $80 \mathrm{~s}(1.6 \%)$ groups accounted for minimum percentage of patients. There was higher proportion of female patients as age increases.

Further analysis showed that the 20s group patients had the highest proportion of connective tissue diseases (Marfan's syndrome) (28.1\%). History of hypertension was present in $63 \%$ patients ages between $40-70$ years old and hypotension on admission was highest in 80 s group (16.7\%, $\mathrm{P}=0.038$ ). (Table 1). The average BMI was 25.6 with the highest 33.1 in 30 s group and lowest 19.5 in 80 s group. There is significantly increased history of stroke( $9.6 \%$ vs $1.9 \%, \mathrm{OR}=5.5,95 \% \mathrm{Cl} 2.6-11.5)$, coronary artery disease (CAD) (5.9\% vs $2.0 \%, \mathrm{OR}=3.1,95 \% \mathrm{Cl} 1.3-7.2)$ in patients over 70 years old. 
Table 1

Baseline demographics and characteristics

\begin{tabular}{|c|c|c|c|c|c|c|c|c|c|}
\hline & Total & $20 s$ & $30 \mathrm{~s}$ & $40 \mathrm{~s}$ & $50 \mathrm{~s}$ & $60 \mathrm{~s}$ & $70 \mathrm{~s}$ & $80 \mathrm{~s}$ & $\begin{array}{l}P \\
\text { value }\end{array}$ \\
\hline Number & 1092 & 32 & 146 & 281 & 286 & 211 & 118 & 18 & \\
\hline Age & $52(62-44)$ & $\begin{array}{l}27(28- \\
25)\end{array}$ & $\begin{array}{l}35.5(38- \\
32)\end{array}$ & $\begin{array}{l}45(47- \\
43)\end{array}$ & $\begin{array}{l}54(56- \\
52)\end{array}$ & $\begin{array}{l}64(66- \\
62)\end{array}$ & $\begin{array}{l}74(76- \\
71)\end{array}$ & $\begin{array}{l}82.5(84.25- \\
80)\end{array}$ & $<0.001$ \\
\hline Male & $\begin{array}{l}816 \\
(74.7 \%)\end{array}$ & $\begin{array}{l}30 \\
(93.8 \%)\end{array}$ & $\begin{array}{l}127 \\
(87.0 \%)\end{array}$ & $\begin{array}{l}244 \\
(86.8 \%)\end{array}$ & $\begin{array}{l}211 \\
(73.8 \%)\end{array}$ & $\begin{array}{l}130 \\
(61.6 \%)\end{array}$ & $\begin{array}{l}64 \\
(54.2 \%)\end{array}$ & $10(55.6 \%)$ & $<0.001$ \\
\hline $\mathrm{BMI}\left(\mathrm{kg} / \mathrm{m}^{2}\right)$ & $25.6 \pm 4.6$ & $25.8 \pm 5.4$ & $27.4 \pm 5.7$ & $26.4 \pm 4.7$ & $25.4 \pm 4.1$ & $24.7 \pm 4.0$ & $24.1 \pm 3.9$ & $23.4 \pm 3.9$ & $<0.001$ \\
\hline Hypertension & $\begin{array}{l}807 \\
(73.9 \%)\end{array}$ & $\begin{array}{l}12 \\
(37.5 \%)\end{array}$ & $\begin{array}{l}95 \\
(65.1 \%)\end{array}$ & $\begin{array}{l}215 \\
(76.5 \%)\end{array}$ & $\begin{array}{l}219 \\
(76.6 \%)\end{array}$ & $\begin{array}{l}162 \\
(76.8 \%)\end{array}$ & $\begin{array}{l}92 \\
(78.0 \%)\end{array}$ & 12 (66.7\%) & $<0.001$ \\
\hline Marfan & $26(2.4 \%)$ & $\begin{array}{l}9 \\
(28.1 \%)\end{array}$ & $4(2.7 \%)$ & $10(3.6 \%)$ & $2(0.7 \%)$ & $1(0.5 \%)$ & $0(0 \%)$ & $0(0 \%)$ & $<0.001$ \\
\hline Diabetes & $40(3.7 \%)$ & $0(0 \%)$ & $3(2.1 \%)$ & $8(2.8 \%)$ & $9(3.1 \%)$ & $11(5.2 \%)$ & $7(5.9 \%)$ & $2(11.1 \%)$ & 0.181 \\
\hline Smoke & $\begin{array}{l}250 \\
(22.9 \%)\end{array}$ & $\begin{array}{l}10 \\
(31.3 \%)\end{array}$ & $\begin{array}{l}34 \\
(23.3 \%)\end{array}$ & $\begin{array}{l}77 \\
(27.4 \%)\end{array}$ & $\begin{array}{l}71 \\
(24.8 \%)\end{array}$ & $\begin{array}{l}40 \\
(19.0 \%)\end{array}$ & $\begin{array}{l}14 \\
(11.9 \%)\end{array}$ & $4(22.2 \%)$ & 0.019 \\
\hline Alcohol & $\begin{array}{l}167 \\
(15.3 \%)\end{array}$ & $3(9.4 \%)$ & $\begin{array}{l}28 \\
(19.2 \%)\end{array}$ & $\begin{array}{l}44 \\
(15.7 \%)\end{array}$ & $\begin{array}{l}54 \\
(18.9 \%)\end{array}$ & $\begin{array}{l}25 \\
(11.8 \%)\end{array}$ & $11(9.3 \%)$ & $2(11.1 \%)$ & 0.098 \\
\hline $\begin{array}{l}\text { End stage kidney } \\
\text { disease }\end{array}$ & $23(2.1 \%)$ & $0(0.0 \%)$ & $4(2.7 \%)$ & $5(1.8 \%)$ & $11(3.8 \%)$ & $0(0.0 \%)$ & $3(2.5 \%)$ & $0(0.0 \%)$ & 0.072 \\
\hline Stroke history & 31 (2.8\%) & $0(0 \%)$ & $1(0.7 \%)$ & $5(1.8 \%)$ & $7(2.4 \%)$ & $5(2.4 \%)$ & $10(8.5 \%)$ & $3(16.7 \%)$ & 0.001 \\
\hline CAD history & $27(2.5 \%)$ & $0(0 \%)$ & $2(1.4 \%)$ & $3(1.1 \%)$ & $5(1.7 \%)$ & $9(4.3 \%)$ & $6(5.1 \%)$ & $2(11.1 \%)$ & 0.020 \\
\hline COPD history & $11(1.0 \%)$ & $0(0 \%)$ & $1(0.7 \%)$ & $2(0.7 \%)$ & $3(0.3 \%)$ & $3(1.4 \%)$ & $2(3.4 \%)$ & $0(0 \%)$ & 0.919 \\
\hline AF history & $10(0.9 \%)$ & $0(0 \%)$ & $1(0.7 \%)$ & $1(0.4 \%)$ & $2(0.7 \%)$ & $3(1.4 \%)$ & $2(1.7 \%)$ & $1(5.6 \%)$ & 0.273 \\
\hline Pain & $\begin{array}{l}1007 \\
(92.3 \%)\end{array}$ & $\begin{array}{l}28 \\
(87.5 \%)\end{array}$ & $\begin{array}{l}134 \\
(91.8 \%)\end{array}$ & $\begin{array}{l}262 \\
(93.2 \%)\end{array}$ & $\begin{array}{l}264 \\
(92.3 \%)\end{array}$ & $\begin{array}{l}194 \\
(91.9 \%)\end{array}$ & $\begin{array}{l}109 \\
(92.4 \%)\end{array}$ & 17 (94.4\%) & 0.936 \\
\hline Chest & $\begin{array}{l}942 \\
(86.3 \%)\end{array}$ & $\begin{array}{l}28 \\
(87.5 \%)\end{array}$ & $\begin{array}{l}125 \\
(85.6 \%)\end{array}$ & $\begin{array}{l}244 \\
(86.8 \%)\end{array}$ & $\begin{array}{l}251 \\
(87.8 \%)\end{array}$ & $\begin{array}{l}179 \\
(84.8 \%)\end{array}$ & $\begin{array}{l}102 \\
(86.4 \%)\end{array}$ & 15 (83.3\%) & 0.965 \\
\hline Back & $\begin{array}{l}433 \\
(39.7 \%)\end{array}$ & $\begin{array}{l}9 \\
(28.1 \%)\end{array}$ & $\begin{array}{l}59 \\
(40.4 \%)\end{array}$ & $\begin{array}{l}110 \\
(39.1 \%)\end{array}$ & $\begin{array}{l}122 \\
(42.7 \%)\end{array}$ & $\begin{array}{l}78 \\
(37.0 \%)\end{array}$ & $\begin{array}{l}50 \\
(42.4 \%)\end{array}$ & $5(27.8 \%)$ & 0.565 \\
\hline Abdominal & $59(5.4 \%)$ & $0(0 \%)$ & $10(6.8 \%)$ & $19(6.8 \%)$ & $12(4.2 \%)$ & $10(4.7 \%)$ & $5(4.2 \%)$ & $3(16.7 \%)$ & 0.197 \\
\hline Leg & $32(2.9 \%)$ & $2(6.3 \%)$ & $5(3.4 \%)$ & $9(3.2 \%)$ & $8(2.8 \%)$ & $6(2.8 \%)$ & $2(1.7 \%)$ & $0(0 \%)$ & 0.848 \\
\hline \multicolumn{10}{|l|}{ Malperfusion } \\
\hline Cerebral & $108(9.9 \%)$ & $0(0 \%)$ & $9(6.2 \%)$ & $26(9.3 \%)$ & $\begin{array}{l}32 \\
(11.2 \%)\end{array}$ & $21(10 \%)$ & $\begin{array}{l}19 \\
(16.1 \%)\end{array}$ & $1(5.6 \%)$ & 0.062 \\
\hline Limb & $\begin{array}{l}165 \\
(15.1 \%)\end{array}$ & $\begin{array}{l}7 \\
(21.9 \%)\end{array}$ & $\begin{array}{l}20 \\
(13.7 \%)\end{array}$ & $\begin{array}{l}49 \\
(17.4 \%)\end{array}$ & $\begin{array}{l}46 \\
(16.1 \%)\end{array}$ & $\begin{array}{l}30 \\
(14.2 \%)\end{array}$ & $\begin{array}{l}12 \\
(10.2 \%)\end{array}$ & $1(5.6 \%)$ & 0.397 \\
\hline Bowel & $47(4.3 \%)$ & $0(0 \%)$ & $4(2.7 \%)$ & $13(4.6 \%)$ & $13(4.5 \%)$ & $10(4.7 \%)$ & $7(5.9 \%)$ & $0(0 \%)$ & 0.666 \\
\hline Myocardial & $52(4.8 \%)$ & $2(6.3 \%)$ & $11(7.5 \%)$ & $10(3.6 \%)$ & $14(4.9 \%)$ & $10(4.7 \%)$ & $5(4.2 \%)$ & $0(0 \%)$ & 0.646 \\
\hline Hypotension & $64(5.9 \%)$ & $1(3.1 \%)$ & $4(2.7 \%)$ & $11(3.9 \%)$ & $20(7.0 \%)$ & $19(9.0 \%)$ & $6(5.1 \%)$ & $3(16.7 \%)$ & 0.040 \\
\hline Pericardial tamponade & $\begin{array}{l}131 \\
(12.0 \%)\end{array}$ & $\begin{array}{l}4 \\
(12.5 \%)\end{array}$ & $5(3.4 \%)$ & $26(9.3 \%)$ & $\begin{array}{l}39 \\
(13.6 \%)\end{array}$ & $\begin{array}{l}34 \\
(16.1 \%)\end{array}$ & $\begin{array}{l}22 \\
(18.6 \%)\end{array}$ & $1(5.6 \%)$ & 0.001 \\
\hline $\begin{array}{l}\text { Coronary artery } \\
\text { involvement }\end{array}$ & $\begin{array}{l}215 \\
(19.7 \%)\end{array}$ & $\begin{array}{l}11 \\
(34.4 \%)\end{array}$ & $\begin{array}{l}28 \\
(19.2 \%)\end{array}$ & $\begin{array}{l}53 \\
(18.9 \%)\end{array}$ & $\begin{array}{l}62 \\
(21.7 \%)\end{array}$ & $\begin{array}{l}39 \\
(18.5 \%)\end{array}$ & $\begin{array}{l}21 \\
(17.8 \%)\end{array}$ & $1(5.6 \%)$ & 0.278 \\
\hline
\end{tabular}

Pain was the main presenting symptom, while chest pain presented as similar among groups. Preoperative malperfusion were present in $34 \%$ of patients with no significant difference among age groups.

\subsection{Operative characteristics}


The duration of surgery, CPB, X-clamp and HCA decreased with advanced age starting from age group of 50 years old. Cannulating both femoral and axillary artery were preferred arterial cannulation approach compared to single femoral or axillary artery. Bentall procedure accounted for a large part of root methods in young age group (43.8\% in 20s) and total arch replacement with FET had a higher rate in age group of $50 \mathrm{~s}(45.8 \%), 60 \mathrm{~s}(41.7 \%)$ than the 70 s $(26.3 \%)$ and 80 s $(16.7 \%)(P<0.001)($ Table 2$)$.

Table 2

Operative data

\begin{tabular}{|c|c|c|c|c|c|c|c|c|c|}
\hline & Total & $20 s$ & $30 \mathrm{~s}$ & $40 s$ & $50 \mathrm{~s}$ & $60 s$ & $70 \mathrm{~s}$ & $80 s$ & $\begin{array}{l}P \\
\text { value }\end{array}$ \\
\hline Number & 1092 & 32 & 146 & 281 & 286 & 211 & 118 & 18 & \\
\hline $\begin{array}{l}\text { Hour from onset } \\
\text { to admission }\end{array}$ & $10(18-6)$ & $10(20-6)$ & $10(18-7)$ & $10(18-7)$ & $9(16-6)$ & $10(20-6)$ & $10(18-6)$ & $9(12-5)$ & 0.374 \\
\hline $\begin{array}{l}\text { Hours from } \\
\text { admission to } \\
\text { surgery }\end{array}$ & $6(12-3)$ & $9(12-3)$ & $6(13-3)$ & $6(14-3)$ & $5(12-3)$ & $5(12-2)$ & $5(10-3)$ & $6(12-3)$ & 0.049 \\
\hline OP duration & $8.0 \pm 2.1$ & $8.2 \pm 2.1$ & $8.2 \pm 2.1$ & $8.3 \pm 2.2$ & $7.8 \pm 2.3$ & $7.7 \pm 1.9$ & $7.7 \pm 1.9$ & $7.1 \pm 1.6$ & $<0.001$ \\
\hline \multicolumn{10}{|l|}{ Cannulation } \\
\hline Ascending & $20(1.8 \%)$ & $2(6.3 \%)$ & $3(2.1 \%)$ & $5(1.8 \%)$ & $3(1.0 \%)$ & $5(2.4 \%)$ & $2(1.7 \%)$ & $0(0 \%)$ & 0.521 \\
\hline Femoral & $\begin{array}{l}231 \\
(21.2 \%)\end{array}$ & $7(21.9 \%)$ & $18(12.3 \%)$ & $36(12.8 \%)$ & $68(23.8 \%)$ & $61(28.9 \%)$ & $33(28.0 \%)$ & $8(44.4 \%)$ & 0.000 \\
\hline Axillary & $\begin{array}{l}233 \\
(21.3 \%)\end{array}$ & $3(9.4 \%)$ & $25(17.1 \%)$ & $64(22.8 \%)$ & $56(19.6 \%)$ & $50(23.7 \%)$ & $30(25.4 \%)$ & $5(27.8 \%)$ & 0.281 \\
\hline Femoral+Axillary & $\begin{array}{l}608 \\
(55.7 \%)\end{array}$ & $20(62.5 \%)$ & $\begin{array}{l}100 \\
(68.5 \%)\end{array}$ & $\begin{array}{l}176 \\
(62.6 \%)\end{array}$ & $\begin{array}{l}159 \\
(55.6 \%)\end{array}$ & $95(45.0 \%)$ & $53(44.9 \%)$ & $5(27.8 \%)$ & 0.000 \\
\hline $\mathrm{HCA}$ & $30.3 \pm 11.1$ & $29.2 \pm 15.6$ & $32.4 \pm 12.6$ & $30.7 \pm 10.8$ & $29.7 \pm 10.8$ & $30.6 \pm 11.1$ & $28.7 \pm 8.5$ & $24.8 \pm 8.7$ & 0.021 \\
\hline CPB & $240.0 \pm 76.1$ & $238.8 \pm 64.4$ & $253.6 \pm 74.2$ & $245.9 \pm 81.4$ & $237.7 \pm 76.7$ & $234.7 \pm 75.9$ & $228.3 \pm 66.9$ & $219.3 \pm 61.1$ & 0.012 \\
\hline X-clamp & $166.8 \pm 61.1$ & $168.5 \pm 47.4$ & $179.8 \pm 59.3$ & $169.8 \pm 72.3$ & $164.1 \pm 57.6$ & $163.1 \pm 57.8$ & $157.8 \pm 49.1$ & $159.7 \pm 59.8$ & 0.049 \\
\hline $\begin{array}{l}\text { Cerebral } \\
\text { perfusion }\end{array}$ & & & & & & & & & $<0.001$ \\
\hline No perfusion & $\begin{array}{l}140 \\
(12.8 \%)\end{array}$ & $7(21.9 \%)$ & $10(6.8 \%)$ & $23(8.2 \%)$ & $43(15.0 \%)$ & 39 (18.5\%) & $14(11.9 \%)$ & $4(22.2 \%)$ & \\
\hline ACP & $\begin{array}{l}886 \\
(81.1 \%)\end{array}$ & $25(78.1 \%)$ & $\begin{array}{l}130 \\
(89.0 \%)\end{array}$ & $\begin{array}{l}250 \\
(89.0 \%)\end{array}$ & $\begin{array}{l}226 \\
(79.0 \%)\end{array}$ & $\begin{array}{l}153 \\
(72.5 \%)\end{array}$ & $91(77.1 \%)$ & $11(61.1 \%)$ & \\
\hline RCP & $66(6.0 \%)$ & $0(0 \%)$ & $6(4.1 \%)$ & $8(2.8 \%)$ & $17(5.9 \%)$ & $19(9.0 \%)$ & $13(11.0 \%)$ & $3(16.7 \%)$ & \\
\hline Root procedure & & & & & & & & & $<0.001$ \\
\hline No & $17(1.6 \%)$ & $2(6.3 \%)$ & $2(1.4 \%)$ & $5(1.8 \%)$ & $3(1.0 \%)$ & $3(1.4 \%)$ & $2(1.7 \%)$ & $0(0 \%)$ & \\
\hline $\begin{array}{l}\text { Root } \\
\text { reconstruction }\end{array}$ & $\begin{array}{l}828 \\
(75.8 \%)\end{array}$ & $13(40.6 \%)$ & 99 (67.8\%) & $\begin{array}{l}213 \\
(75.8 \%)\end{array}$ & $\begin{array}{l}214 \\
(74.8 \%)\end{array}$ & $\begin{array}{l}174 \\
(82.5 \%)\end{array}$ & $\begin{array}{l}101 \\
(85.6 \%)\end{array}$ & $14(77.8 \%)$ & \\
\hline Bentall & $\begin{array}{l}228 \\
(20.9 \%)\end{array}$ & $14(43.8 \%)$ & $38(26.0 \%)$ & $60(21.4 \%)$ & $68(23.8 \%)$ & $30(14.2 \%)$ & $14(11.9 \%)$ & $4(22.2 \%)$ & \\
\hline VSRR & $19(1.7 \%)$ & $3(9.4 \%)$ & $7(4.8 \%)$ & $3(1.1 \%)$ & $1(0.3 \%)$ & $4(1.9 \%)$ & $1(0.8 \%)$ & $0(0 \%)$ & \\
\hline Arch procedure & & & & & & & & & $<0.001$ \\
\hline Sub-arch & $\begin{array}{l}211 \\
(19.9 \%)\end{array}$ & $7(21.9 \%)$ & $18(12.3 \%)$ & 40 (14.2\%) & 49 (17.1\%) & 47 (22.3\%) & 45 (38.1\%) & $7(38.9 \%)$ & \\
\hline Total arch + FET & $\begin{array}{l}515 \\
(47.1 \%)\end{array}$ & 19 (59.4\%) & $88(60.3 \%)$ & $\begin{array}{l}156 \\
(55.5 \%)\end{array}$ & $\begin{array}{l}131 \\
(45.8 \%)\end{array}$ & 88 (41.7\%) & 31 (26.3\%) & $3(16.7 \%)$ & \\
\hline Arch stent & $\begin{array}{l}361 \\
(33.0 \%)\end{array}$ & $6(18.8 \%)$ & $40(27.4 \%)$ & $85(30.2 \%)$ & $\begin{array}{l}106 \\
(37.1 \%)\end{array}$ & $74(35.1 \%)$ & $41(34.7 \%)$ & $8(44.4 \%)$ & \\
\hline
\end{tabular}




\subsection{Immediate postoperative outcomes}

One hundred and fifty-four patients (14.1\%) died within 30 days after surgery, $93(60.4 \%)$ from circulatory failure, 21 (13.6\%) neurological complications, $15(9.7 \%)$ aortic rupture, $13(8.4 \%)$ respiratory failure or other reasons, $12(7.8 \%)$ gastrointestinal bleeding or ischemia, (Table 3). Group 80 s had significantly higher mortality than group 70 s ( $33.3 \%$ vs $18.6 \%, p=0.016$ ) (Figure $2 a)$. A decreasing trend of mortality rate in 70 s and 80 s group was shown with year (Figure 2b). Age was related to postoperative complications. The stroke rates (died and not died) were $8.5 \%, 5.1 \%$ and $5.6 \%$ in $60 \mathrm{~s}, 70 \mathrm{~s}$ and $80 \mathrm{~s}$ group respectively. Among patients succumbed in the 80 year group, there were significant high rate of $\mathrm{GI}$ bleeding and ischemia ( $16.7 \%$ and $11.1 \%$, respectively) compared with other groups of $50 \mathrm{~s}, 60 \mathrm{~s}$ and $70 \mathrm{~s}(1.4 \%, 0 \%, 0.8 \%$, respectively ). The 70 s group had higher duration of ICU stay when compared with other groups (20s group: $p=0.048$, 30 s group: $p=0.047$, 50 s group: $p=0.011$ ); however, the duration of hospital stay showed no significant difference in all age groups. There was no significant difference in mechanical ventilation, reintubation, tracheotomy, neurological complications, renal complications and re-exploration. The 20s group showed lowest postoperative neurological and gastrointestinal complications as they presented at admission. (Table 3).

Table 3

Postoperative data

\begin{tabular}{|c|c|c|c|c|c|c|c|c|c|}
\hline & Total & $20 \mathrm{~s}$ & $30 \mathrm{~s}$ & $40 \mathrm{~s}$ & $50 \mathrm{~s}$ & $60 \mathrm{~s}$ & $70 \mathrm{~s}$ & $80 \mathrm{~s}$ & $\begin{array}{l}\mathrm{P} \\
\text { value }\end{array}$ \\
\hline Number & 1092 & 32 & 146 & 281 & 286 & 211 & 118 & 18 & \\
\hline 30 day-mortality & $\begin{array}{l}154 \\
(14.1 \%)\end{array}$ & $5(15.6 \%)$ & 11 (7.5\%) & $\begin{array}{l}33 \\
(11.7 \%)\end{array}$ & $\begin{array}{l}41 \\
(14.3 \%)\end{array}$ & $\begin{array}{l}36 \\
(17.1 \%)\end{array}$ & $\begin{array}{l}22 \\
(18.6 \%)\end{array}$ & $6(33.3 \%)$ & 0.016 \\
\hline $\begin{array}{l}\text { Mechanical } \\
\text { ventilation }\end{array}$ & $56.2 \pm 79.1$ & $71.6 \pm 130.2$ & $61.2 \pm 90.4$ & $55.2 \pm 69.6$ & $57.5 \pm 93.8$ & $52.9 \pm 63.3$ & $49.3 \pm 51.0$ & $67.6 \pm 80.4$ & 0.808 \\
\hline Reintubation & $70(6.4 \%)$ & $4(12.5 \%)$ & $6(4.1 \%)$ & $14(5.0 \%)$ & $23(8.0 \%)$ & $12(5.7 \%)$ & $11(9.3 \%)$ & $0(0 \%)$ & 0.199 \\
\hline Tracheotomy & $44(4.0 \%)$ & $2(6.3 \%)$ & $4(2.7 \%)$ & $11(3.9 \%)$ & $10(3.5 \%)$ & $7(3.3 \%)$ & $10(8.5 \%)$ & $0(0 \%)$ & 0.216 \\
\hline $\mathrm{ICH}$ & $9(0.8 \%)$ & $0(0 \%)$ & $1(0.7 \%)$ & $1(0.4 \%)$ & $5(1.7 \%)$ & $1(0.5 \%)$ & $1(0.8 \%)$ & $0(0 \%)$ & 0.609 \\
\hline Stroke & $59(5.4 \%)$ & $0(0 \%)$ & $3(2.1 \%)$ & $14(5.0 \%)$ & $17(5.9 \%)$ & $18(8.5 \%)$ & $6(5.1 \%)$ & $1(5.6 \%)$ & 0.155 \\
\hline Paraplegia & $23(2.1 \%)$ & $0(0 \%)$ & $4(2.7 \%)$ & $9(3.2 \%)$ & $7(2.4 \%)$ & $2(0.9 \%)$ & $1(0.8 \%)$ & $0(0 \%)$ & 0.489 \\
\hline GI bleeding & $13(1.2 \%)$ & $0(0 \%)$ & $1(0.7 \%)$ & $4(1.4 \%)$ & $4(1.4 \%)$ & $0(0 \%)$ & $1(0.8 \%)$ & $3(16.7 \%)$ & 0.000 \\
\hline Limb ischemia & $13(1.2 \%)$ & $0(0 \%)$ & $1(0.7 \%)$ & $5(1.8 \%)$ & $2(0.7 \%)$ & $5(2.4 \%)$ & $0(0 \%)$ & $0(0 \%)$ & 0.396 \\
\hline Bowel ischemia & $16(1.5 \%)$ & $0(0 \%)$ & $3(2.1 \%)$ & $3(1.1 \%)$ & $3(1.0 \%)$ & $2(0.9 \%)$ & $3(2.5 \%)$ & $2(11.1 \%)$ & 0.025 \\
\hline Surgical site infection & $37(3.4 \%)$ & $2(6.3 \%)$ & $3(2.1 \%)$ & $10(3.6 \%)$ & $9(3.1 \%)$ & $11(5.2 \%)$ & $2(1.7 \%)$ & $0(0 \%)$ & 0.484 \\
\hline Acute renal failure & $\begin{array}{l}339 \\
(31.0 \%)\end{array}$ & $10(31.3 \%)$ & $\begin{array}{l}50 \\
(34.2 \%)\end{array}$ & $\begin{array}{l}89 \\
(31.7 \%)\end{array}$ & $\begin{array}{l}97 \\
(33.9 \%)\end{array}$ & $\begin{array}{l}56 \\
(26.5 \%)\end{array}$ & $\begin{array}{l}31 \\
(26.3 \%)\end{array}$ & $6(33.3 \%)$ & 0.525 \\
\hline CRRT & $\begin{array}{l}127 \\
(11.6 \%)\end{array}$ & $2(6.3 \%)$ & $14(9.6 \%)$ & $\begin{array}{l}35 \\
(12.5 \%)\end{array}$ & $28(9.8 \%)$ & $\begin{array}{l}27 \\
(12.8 \%)\end{array}$ & $\begin{array}{l}16 \\
(13.6 \%)\end{array}$ & $5(27.8 \%)$ & 0.246 \\
\hline Reexploration & $64(5.8 \%)$ & $0(0 \%)$ & $6(4.1 \%)$ & $14(5.0 \%)$ & $26(9.1 \%)$ & $11(5.2 \%)$ & $6(5.1 \%)$ & $0(0 \%)$ & 0.119 \\
\hline ICU stay (days) & $5(8-3)$ & $4(6-3)$ & $5(7-3)$ & $6(10-3)$ & $5(8-3)$ & $5(9.5-3)$ & $6(10-4)$ & $5(12-3)$ & 0.046 \\
\hline Hospital stay (days) & $20.8 \pm 13.1$ & $21.9 \pm 11.4$ & $20.5 \pm 10.0$ & $21.7 \pm 13.2$ & $19.5 \pm 11.5$ & $21.8 \pm 14.5$ & $20.5 \pm 17.2$ & $14.4 \pm 10.8$ & 0.236 \\
\hline
\end{tabular}

\subsection{Follow-up}

927 (84.9\%) patients completed followed up and the median follow-up was 17 months (1-102months). The 17-month survival rate was $82 \%$. Thirtyeight discharged patients died during follow-up, $1(3.1 \%)$ in 20 s group, $4(12.5 \%)$ in 30 s group, $8(25 \%)$ in 40 s group, $7(21.9 \%)$ in 50 s group, $9(28.1 \%)$ in 60 s group, $8(25 \%)$ in 70 s group, and $1(3.1 \%)$ in 80 s group. Figure 3 a shows the mortality associated with age, the general tendency is that mortality increases with increasing age, $15.6 \%$ in 20 s group, $7.5 \%$ in 30 s group, $11.7 \%$ in 40 s group, $14.3 \%$ in 50 s group, $17.1 \%$ in 60 s group, $18.6 \%$ in 70 s group, and $33.3 \%$ in 80 s group; however, the mortality of patients over 70 years old decreased over these years while the ratio of these patients remains relatively stable. Among the patients who died, 10 (26.3\%) from aortic rupture, 5(13.2\%) from neurological complications, and 2(5.3\%) patients died for stent leakage. 137 patients had readmissions, of whom 34 (24.8\%) patients had thoracoabdominal aortic dissection/aneurysm which is the leading cause of readmission. Figure $3 \mathrm{~b} / 3 \mathrm{c}$ shows the ratio of patients readmission for recurrence of aortic dissection, the tendency of readmission for abdominal aortic dissection decreased with age. 


\section{Discussion}

The average age of aTAAD patients was significantly younger in China, the results from Sino-RAD was 50.5 years[12] and 52 years in our center's previous reports[5][13]. In this study, we found the median age of patients of aTAAD is 52. Furthermore, there was a significant increasing number of patients underwent aTAAD in the older patients at their 70s and 80s. There was also significant increase of aTAAD repair in patients aging 70 years and older. The underlining reasons maybe multi-factorial. One of the main reasons is the increasing awareness of aTAAD among the public and emergency room physicians particularly since the introduction of our aTAAD refereral program (6hr life circle); the second is attributed to the improvement of surgical successful rate of aTAAD surgery; and 3rd maybe related to the recent increase of national and regional healthcare coverage[14] and especially in the second half of the study period(2016-2019). For older patients with aTAAD, the optimal treatment strategy is in debate depending on the risk and benefit ratio and the upper age limit is unknown. A study by Trimarchi et al using IRAD data showed that patients older than 70 years old received higher rates of medical therapy than those of surgical repair $(28.6 \%$ vs $10.9 \%$; $<<0.0001)$, and there was no difference in survival between the two treatment strategies (55.8\% vs $53.8 \%$; $p=0.32)$ [1]. Our results are consistent with the above findings. The increasing risks and lower predictable late survival rate contributed to the lower proportion of open surgery. Many studies have shown that advanced age is related to poor postoperative survival, the long-term survival and the quality of daily life[15-17]. The poor outcome in patients of advanced age may have deterred the Septuagenarian and Octogenarian from undergoing extensive total arch surgery because there is no better alternative treatment such as endovascular repair.

Our present study also demonstrated that the proportion of patients who received surgical treatment over 70 years old remained relatively stable in last decade in our center. The higher 30 mortality in this group of patients suggested the negative impact of surgery on the postoperative recovery of patients with advanced age. In addition, the favorable long term survival indicated that the simple or less extensive arch repair is the preferred surgery for patients over 70 years old. This finding is also corroborated by other studies[2][18].

Chest pain is the common clinical presentations of aTAAD for younger patients; however, the main etiology of aTAAD for patients in their 20s is connective tissue disorders, such as Marfan syndrome while history of hypertension is more common in patients of aging 30,40 and 50 s. Compared to the patients older than 70 years, the surgical strategies are totally different. More extensive surgical methods are applied for younger patients in order to avoid re-intervention because of aortic events[19-23]. Our study demonstrated that the recurrence rate of aTAAD was significantly lower in younger patients between 30 to 50 years old. The patients of 20 s group had the highest recurrent AD, consistant with the findings in Marfan's syndrome paitents reported by Isselbacher et al. from the IRAD data[24]. The ratio of readmission for aortic dissection is also decreased with age. Because older patients had more complications than younger patients. These complications not only affect the time and strategies of surgery, but also affect the outcomes of the patients. And they had to have readmission to deal with the complications after surgery. On the contrary, the 20s group had readmission most likely to deal with the recurrent aortic dissection. Therefore, extensive surgery strategy with higher surgical risk could not lower late recurrence and re-intervention.

The mode of the age was in the 40s group, these patients were at the middle age of their life. It was necessary to pay more attention to their long term follow up and the quality of life. In the next years, we would focus on their changes and show what would happen to these post-operative aortic dissection patients. For the increasing number of hypertension patients in China, it was meaningful to know whether the 40 s group patients could totally recovery from the emergency surgery and go back to the society.

\section{Limitations}

First, the retrospective study has its design limitation. Data were collected retrospectively so there are defects like incomplete, missing or inaccurate to report the event. The long-term survival rate would be underestimate as the follow-up interval is large and the follow-up time of some patients is shorter than one year. Second, the data obtained are of a single center and therefore could not represent the whole population. Third, as the number of patients in 20 s and 80 s group being limited, there is a need for further studies.

\section{Conclusions}

Age is a major impact factor for aTAAD surgery. Old patients presented more comorbidities before surgery, the mortality and complications rate were significantly higher even with less invasive and conservative surgical therapy. But the favorable long term survival indicated that the simple or less extensive arch repair is the preferred surgery for patients over 70 years old.

\section{Abbreviations}

aTAAD: acute type A aortic dissection

$A D$ : aortic dissection

\section{Declarations}




\section{Ethical approval and consent to participate}

The current study was approved by the institutional review board of Nanjing Drum Tower Hospital (2020-185-01) and adhered to the tenets of the Declaration of Helsinki.

\section{Consent for publication}

Not applicable.

\section{Availability of data and materials}

Data sharing is not applicable to this article as no datasets were generated or analysed during the current study.

\section{Competing interests}

The authors declare that they have no conflicts of interest.

\section{Funding}

This work was supported by the National Natural Science Foundation of China (Nos. 81970401, 8210021727) and Jiangsu Provincial Key Medical Discipline (ZDXKA2016019).

\section{Authors' contributions}

J-XW and Y-XX designed the study. J-XW, Y-XX, X-YZ and ZC collected and analyzed the data together. J-XW, Y-XX, H-SC and QZ drafted the article and submitted the manuscript. D-JW and J-ZQ supervised this study. All authors read the final version of this article and approved for publication.

\section{Acknowledgements}

Not applicable.

\section{References}

1. S. Trimarchi, K.A. Eagle, C.A. Nienaber, V. Rampoldi, F.H.W. Jonker, C. De Vincentiis, A. Frigiola, L. Menicanti, T. Tsai, J. Froehlich, A. Evangelista, D. Montgomery, E. Bossone, J. V. Cooper, J. Li, M.G. Deeb, G. Meinhardt, T.M. Sundt, E.M. Isselbacher, Role of age in acute type A aortic dissection outcome: Report from the International Registry of Acute Aortic Dissection (IRAD), J. Thorac. Cardiovasc. Surg. 140 (2010) $784-789$. https://doi.org/10.1016/j.jtcvs.2009.11.014.

2. H. Kondoh, H. Satoh, T. Daimon, Y. Tauchi, J. Yamamoto, K. Abe, H. Matsuda, Outcomes of limited proximal aortic replacement for type A aortic dissection in octogenarians, J. Thorac. Cardiovasc. Surg. 152 (2016) 439-446. https://doi.org/10.1016/j.jtcvs.2016.03.093.

3. E. Suenaga, M. Sato, H. Fumoto, Ascending aortic replacement for acute type A aortic dissection in octogenarians, Gen. Thorac. Cardiovasc. Surg. 64 (2016) 138-143. https://doi.org/10.1007/s11748-015-0613-0.

4. B. Rylski, M. Suedkamp, F. Beyersdorf, B. Nitsch, I. Hoffmann, M. Blettner, E. Weigang, Outcome after surgery for acute aortic dissection type A in patients over 70 years: Data analysis from the German Registry for Acute Aortic Dissection Type A (GERAADA), Eur. J. Cardio-Thoracic Surg. 40 (2011) 435-440. https://doi.org/10.1016/j.ejcts.2010.11.073.

5. A.L. Axtell, Y. Xue, J.Z. Qu, Q. Zhou, J. Pan, H. Cao, T. Pan, A.S. Jassar, D. Wang, T.M. Sundt, D.E. Cameron, Type A aortic dissection in the East and West: A comparative study between two hospitals from China and the US, J. Card. Surg. 35 (2020) 2168-2174. https://doi.org/10.1111/jocs.14766.

6. S.W. Chen, Y. Chen, W.G. Ma, Y.L. Zhong, Z.Y. Qiao, Y.P. Ge, C.N. Li, J.M. Zhu, L.Z. Sun, Limited vs. extended repair for acute type I aortic dissection: long-term outcomes over a decade in Beijing Anzhen Hospital, Chin. Med. J. (Engl). 134 (2021) 986-988. https://doi.org/10.1097/CM9.0000000000001416.

7. Y. Xue, J. Pan, H. Cao, F. Fan, X. Luo, M. Ge, Y. Chen, D. Wang, Q. Zhou, Different aortic arch surgery methods for type A aortic dissection: Clinical outcomes and follow-up results, Interact. Cardiovasc. Thorac. Surg. 31 (2020) 254-262. https://doi.org/10.1093/icvts/ivaa095.

8. J. Pan, Q.G. Li, Q. Zhou, Q. Wang, Z. Wu, D.J. Wang, Repair of acute type A aortic dissections using open replacement with triple-branched stent grafts, Ann. Thorac. Surg. 96 (2013) 559-562. https://doi.org/10.1016/j.athoracsur.2013.04.020.

9. Q. Zhou, Y. Xue, H. Cao, J. Pan, Q. Wang, F. Fan, D. Wang, Novel arch fenestrated stent graft for acute Stanford Type A aortic dissection with open antegrade implantation, Interact. Cardiovasc. Thorac. Surg. 26 (2018) 369-375. https://doi.org/10.1093/icvts/ivx335.

10. Y. Xue, Q. Zhou, J. Pan, H. Cao, F. Fan, X. Zhu, H. Chong, D. Wang, Root reconstruction for proximal repair in acute type A aortic dissection, J. Thorac. Dis. 11 (2019) 4708-4716. https://doi.org/10.21037/jtd.2019.10.62.

11. Y. Xue, Q. Zhou, J. Pan, H. Cao, F. Fan, X. Zhu, D. Wang, “Double Jacket Wrapping” Root Reconstruction for Acute Type A Aortic Dissection, Ann. Thorac. Surg. 110 (2020) 1060-1062. https://doi.org/10.1016/j.athoracsur.2020.03.081.

Page $8 / 11$ 
12. W. Wang, W. Duan, Y. Xue, L. Wang, J. Liu, S. Yu, D. Yi, Clinical features of acute aortic dissection from the Registry of Aortic Dissection in China, J. Thorac. Cardiovasc. Surg. 148 (2014) 2995-3000. https://doi.org/10.1016/j.jtcvs.2014.07.068.

13. Y. Lu, Y. Xue, H. Zhang, W. Xie, W. Zhao, D. Wang, Q. Zhou, Management strategy of Type A aortic dissection in a developing center from China: 16 Years experiences, J. Thorac. Dis. 12 (2020) 6780-6788. https://doi.org/10.21037/jtd-20-1866.

14. Y. Zhou, H. Wushouer, D. Vuillermin, B. Ni, X. Guan, L. Shi, Medical insurance and healthcare utilization among the middle-aged and elderly in China: Evidence from the China health and retirement longitudinal study 2011, 2013 and 2015, BMC Health Serv. Res. 20 (2020) 1-9. https://doi.org/10.1186/s12913-020-05522-w.

15. A. Omura, H. Matsuda, H. Minami, H. Nakai, S. Henmi, H. Murakami, M. Yoshida, N. Mukohara, Early and Late Outcomes of Operation for Acute Type A Aortic Dissection in Patients Aged 80 Years and Older, Ann. Thorac. Surg. 103 (2017) 131-138. https://doi.org/10.1016/j.athoracsur.2016.05.046.

16. R.H. Mehta, P.T. O’Gara, E. Bossone, C.A. Nienaber, T. Myrmel, J. V. Cooper, D.E. Smith, W.F. Armstrong, E.M. Isselbacher, L.A. Pape, K.A. Eagle, D. Gilon, Acute type A aortic dissection in the elderly: Clinical characteristics, management, and outcomes in the current era, J. Am. Coll. Cardiol. 40 (2002) 685-692. https://doi.org/10.1016/S0735-1097(02)02005-3.

17. E. Neri, T. Toscano, M. Massetti, G. Capannini, E. Carone, E. Tucci, F. Diciolla, S. Scolletta, R. Morello, C. Sassi, Operation for acute type A aortic dissection in octogenarians: Is it justified?, J. Thorac. Cardiovasc. Surg. 121 (2001) 259-267. https://doi.org/10.1067/mtc.2001.112205.

18. T. Igarashi, Y. Sato, H. Satokawa, S. Takase, H. Wakamatsu, Y. Seto, M. Iwai-Takano, T. Fujimiya, H. Shinjo, H. Yokoyama, The results of an entryoriented strategy for acute type A aortic dissection in octogenarians: An 18-year experience, Eur. J. Cardio-Thoracic Surg. 58 (2020) $949-956$. https://doi.org/10.1093/EJCTS/EZAA195.

19. A.S. Abdelhameed, F. Xin, X. Wei, Early mortality in patients who received extensive surgical management for acute type a aortic dissection analysis of 452 consecutive cases from a single-center experience, Brazilian J. Cardiovasc. Surg. 35 (2020) 521-529. https://doi.org/10.21470/1678-9741-2019-0258.

20. B. Dib, P.C. Seppelt, R. Arif, A. Weymann, G. Veres, B. Schmack, C.J. Beller, A. Ruhparwar, M. Karck, K. Kallenbach, Extensive aortic surgery in acute aortic dissection type A on outcome - Insights from 25 years single center experience, J. Cardiothorac. Surg. 14 (2019) 1-10. https://doi.org/10.1186/s13019-019-1007-7.

21. J.B. Kim, Extensive aortic repair in acute aortic dissection: Not much bang for the buck?, J. Thorac. Cardiovasc. Surg. 156 (2018) $949-950$. https://doi.org/10.1016/j.jtcvs.2018.04.012.

22. B. Rylski, J.E. Bavaria, F. Beyersdorf, E. Branchetti, N.D. Desai, R.K. Milewski, W.Y. Szeto, P. Vallabhajosyula, M. Siepe, F.A. Kari, Type A aortic dissection in marfan syndrome: Extent of initial surgery determines long-term outcome, Circulation. 129 (2014) 1381-1386. https://doi.org/10.1161/CIRCULATIONAHA.113.005865.

23. Y. Yan, L. Xu, H. Zhang, Z.Y. Xu, X.Y. Ding, S.W. Wang, X. Xue, M.W. Tan, Proximal aortic repair versus extensive aortic repair in the treatment of acute type A aortic dissection: A meta-analysis, Eur. J. Cardio-Thoracic Surg. 49 (2016) 1392-1401. https://doi.org/10.1093/ejcts/ezv351.

24. E.M. Isselbacher, C.L.L. Cardenas, M.E. Lindsay, Hereditary influence in thoracic aortic aneurysm and dissection, Circulation. 133 (2016) 25162528. https://doi.org/10.1161/CIRCULATIONAHA.116.009762.

\section{Figures}
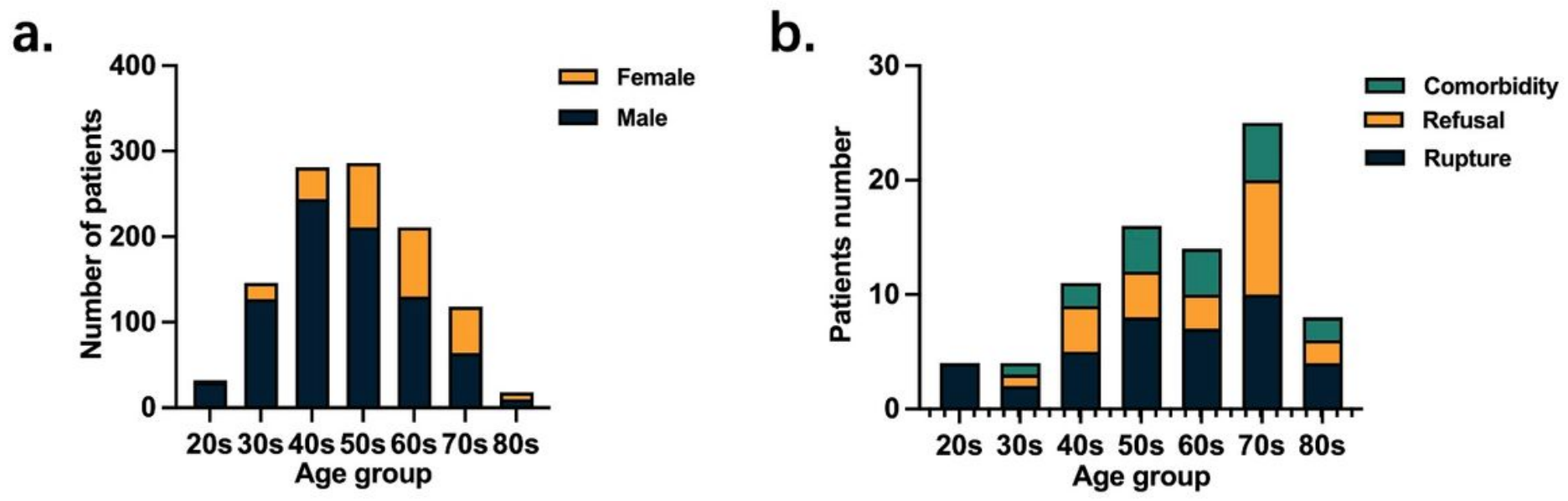

Figure 1 
a. Number and sex ratio of patients in different age groups of our center; $b$. The reason for non-surgery after admission. The main reason was a rupture.

mortality

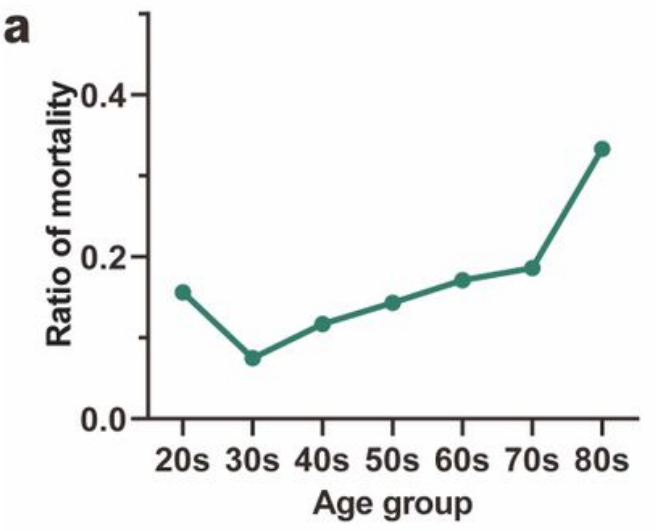

Ratio and mortality of $\geq 70$ s in each year



Figure 2

a. Ratio of mortality in different age groups. b. Ratio and mortality of $\geq 70$ years old patients each year, and the overall mortality fluctuation from 2011-2019.

a.

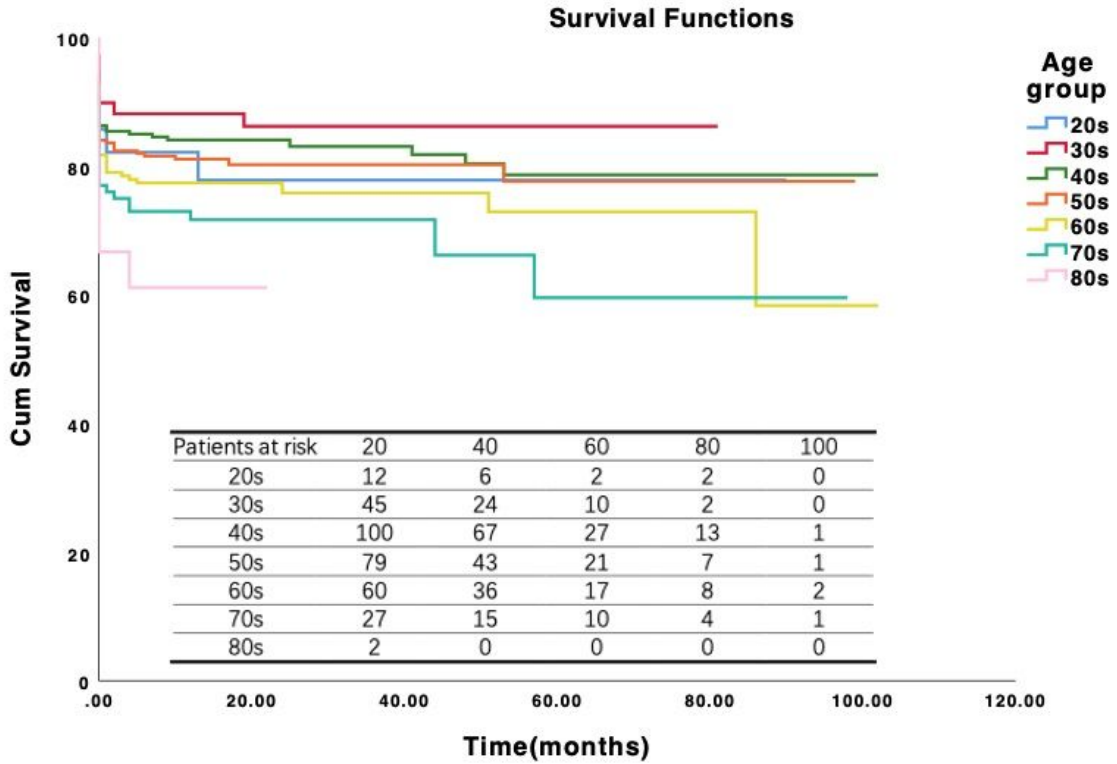

b.

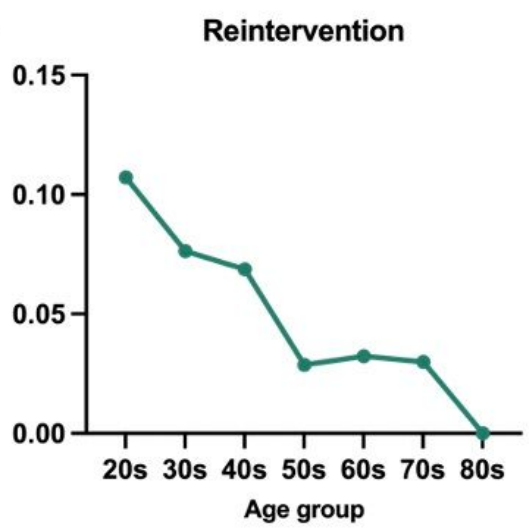

C. Ratio of readmission for aortic dissection

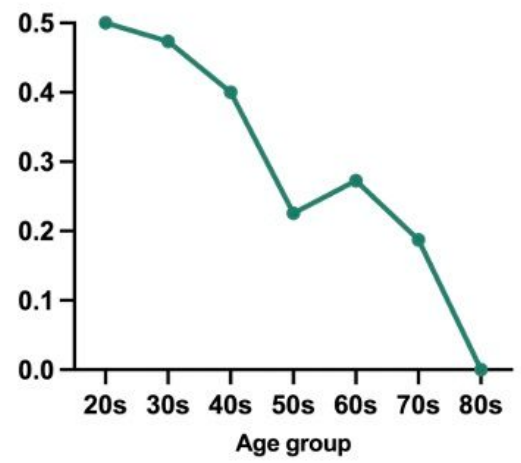

Figure 3 
a. Survival functions of different age groups. b. The reintervention rate of different age groups. c. The ratio of readmission for aortic dissection 